\title{
Effective Active Noise Control algorithm for gain and power constraints with limiting factor using adaptive filter
}

\author{
Mr. V. Saravanan ${ }^{1}$, Mr. N. Sunil Vignesh ${ }^{2}$ \\ ${ }^{I}$ (Electronics and Communication Engineering, Nandha Engineering college, India) \\ ${ }^{2}$ (Electronics and Communication Engineering, Nandha Engineering college, India)
}

\begin{abstract}
In this paper, a new algorithm with multi constraints that can be used in active noise control is presented. This can be used to maintain the particular system power budget and prevent over driving. The filter used for the constraints is frequency domain of least mean square algorithm. A limiting factor is used in the algorithm to reduce the effect of the filter response at other frequencies. The algorithm can be either used in gain or output power constraint. The algorithm shows the better convergence rate than the existing algorithms. The finding of correct convergence point can be done through the secondary path.
\end{abstract}

Keywords: Active Noise Control, Adaptive filter, Convergence, Limiting algorithm, Multi constrained filter

\section{Introduction}

In this paper, we present a new algorithm with multi constraints that can be used in active noise control. This can be used to maintain the particular system power budget. The filter used for the constraints is frequency domain of least mean square algorithm. A limiting factor is used in the algorithm to reduce the effect of the filter response at other frequencies. The algorithm can be either used in gain or output power constraint. The gain and power limiting with the leaky LMS and the Constraint steepest descent (CSD) algorithm were implemented. The power controlled and gain controlled algorithm termed controlled least distraction (CLD) is proposed in this paper. The new algorithm shows the better convergence rate than the existing algorithms. The finding of correct convergence point can be done through the secondary path with multiple limitation handling.

\section{Literature Survey}

In Non Linear Active Noise control using NARX technique, it has advantage of reduced model size and robustness. But the Computational complexity is more in this technique.

In FGPA implementation of a modular active noise control system, high speed programmable gate array active noise control system with on line secondary path modelling is used. It has advantage of fast design and fast convergence with capability to expand the four adaptive filter and data bus. But the system needs $66.7 \mathrm{~ns}$ to execute iteration and the proposed design needs 190 iterations for an acceptable convergence.

In an improved active control system without secondary path model, strict positive real property of the filtered-x LMS algorithm is employed. It has the merit of finding the right phase compensating amount of secondary path automatically. But it is limited to +90 bound property and it doesn't work when the phase shift of secondary path close to +90

In the delay less sub band an adaptive filtering algorithm for Active Noise Control systems, the UDFTM-based adaptive sub band filtering method has been used. It improves the performance and reduces computational complexity, but causes delay and side-lobes of the prototype filter.

In FXLMS based Narrowband Active Noise Control with a secondary path modelling, steady state expression for the mean square error as well as the residual noise power is developed. It has limitation of extensive simulation.

Adaptive Filtering with averaging based filtered reference algorithm is the technique being used in New Structure For Feed forward Active Noise Control System with improved Online Secondary path Modelling. In this secondary path modelling error can be reduced at a faster convergence rate but it takes Long memory and hence poor tracking properties.

In the Project Review of DSP Algorithms for Active Noise Control FXLMS algorithm has been used with multiple-channel, cases for controlling the noise field but it doesn't recognize varying signals and its sensitive.

In Narrowband Active Noise Control, filtered-X LMS (FXLMS) algorithm has been used. In this model lower the noise reduction can be achieved but larger phase error can occur.

In the Active Noise Control System for MR noise with the Implementation of ANC system by digital signal processor, Feedback is used in the ANC system which reduces the MR noise effectively but the system has a fatal defect. 
In Adaptive Noise Cancellation Algorithm for Speech Processing, Normalized difference LMS (NDLMS) is used which results in better performance such as lower level in excess MSE and maladjustment. It slows the varying signal and less sensitivity.

In the Method For Active Noise Control Systems With Online Acoustic-Feedback-Path modelling, FxLMS algorithm based noise control filter, a variable step Microphone Secondary E-Microphone size (VSS) LMS as it removes the disturbance from the desired response of FBPM filter but it need continuous adjustments during the ANC system.

CLD algorithm appears to be the best choice as a controlling filter, researchers tend to improve performance of ANC systems by enhancing and modifying this algorithm. In this paper, modification is done in the existing CSD algorithm that provides a new structure for improving the multi-constraints and convergence rate. The secondary signal $y(n)$ is dynamic threshold by Wavelet transform to improve multi-constraints. The convergence rate is improved by dynamically varying the step size of error signal. The proposed algorithm called constrained Minimal Disturbance uses both data and correction terms to find the updated values of the tap weights of the ANC controller. The computer simulations are conducted for single-channel feed forward ANC system. It is shown that the proposed algorithm gives fast convergence as compared with the previous algorithm and achieves better performance in the presence of the measurement noise.

\section{Gain Constrained Algorithm}

The new algorithm will minimize the frequency domain weight change in each individual frequency in each block and the weight change is subject to the condition of a posterior filter convergence which analysed in the frequency domain. In most of the gain-constrained applications, the algorithm will additionally add a limiting factor based on the amount of magnitude violation above a maximum constraint value In steady state (after convergence), the successive weight values are expected to be approximately the same for a small convergence coefficient step size. Therefore, as long as the constraint was satisfied in the previous iteration, the limiting factor is set to 0 . However, if the magnitude of the filter weight exceeds the constraint value, then the limiting factor is scaled in proportion to the constraint violation. The algorithm will have the following behaviour such as, if the constraint is violated, the CLD algorithm will reduce the magnitude of the adaptive filter frequency response in proportion to the level of constraint violation. The algorithm also normalizes the weight update in a manner similar to the

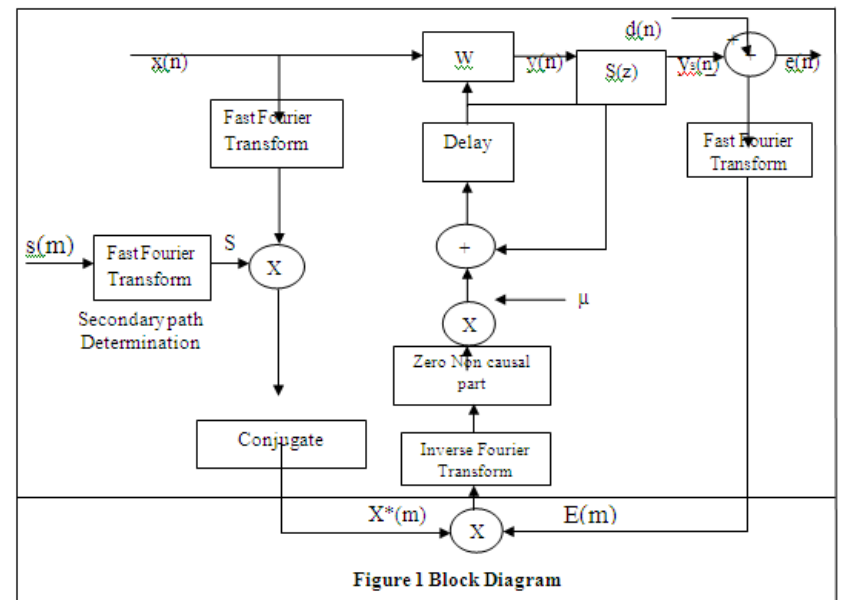

Normalized-LMS with leakage. The amount of leakage is dependent on the level of constraint

violation. The algorithm scales the weight update by the inverse of secondary path frequency response, resulting in a faster convergence in regions corresponding to valleys (low magnitude response) in the secondary path.

Weight vector given by,

$w(m)=\left[w_{\mathrm{o}}(n) w_{1}(n) \ldots \mathrm{w}_{N-1}(n)\right]^{\mathrm{T}}$

Input vector given by,

Error vector given by,

$$
x(m)=[x(n) x(n-1) \ldots x(n-N+1)]
$$

$$
e(m)=[e(n) e(n-1) \ldots e(n-N+1)]^{\mathrm{T}}
$$

Taking FFT,

$$
X(m)=F F T\left\{\left[x^{\mathrm{T}}(n-N) x^{\mathrm{T}}(n)\right]^{\mathrm{T}}\right\}
$$


Change in in weight is given by,

$$
E(m)=F F T\left\{\left[0 \quad e^{\mathrm{T}}(n)\right]^{\mathrm{T}}\right\}
$$

Output frequency domain is given by,

$$
\delta W(m+1)=W(m+1)-W(m)
$$

The limiting factor for the constraint is,

$$
D(m)=S(m) W(m+1) W(m)
$$

$$
\begin{gathered}
|W(m+1)| \leq \sqrt{C} \\
{[\mathrm{D}(\mathrm{m})-\mathrm{S}(\mathrm{m}) \mathrm{W}(\mathrm{m}) \mathrm{X}(\mathrm{m})]+\alpha \mathrm{D}(\mathrm{m})-\frac{1}{2} \lambda|| \mathrm{S}(\mathrm{m})\left\|^{2}|| \mathrm{X}(\mathrm{m})\right\|^{2}=0}
\end{gathered}
$$

$$
\lambda=\frac{2[E(m)+\alpha \mathrm{D}(\mathrm{m})]}{S(m)^{2} X(m)^{2}}
$$

Where $\lambda, \alpha$ are the Lagrange multipliers,

$$
\begin{aligned}
\mathrm{W}(\mathrm{m}+1) & =\frac{1}{[1+\alpha]}\left[\mathrm{W}(\mathrm{m})+\mu \frac{[E(m)+\alpha \mathrm{D}(\mathrm{m})]}{S(m)^{2} X(m)} S^{*}(\mathrm{~m}) X^{*}(\mathrm{~m})\right] \\
\alpha & \left.=2 \alpha\left[\mathrm{W}(\mathrm{m})^{2}-\mathrm{C}\right)\right]
\end{aligned}
$$

$\mu$ is convergence step size coefficient

$$
\begin{gathered}
D(m)=S(m) W(m+1) W(m) \\
\mathrm{W}(\mathrm{m}+1)=\left(\frac{1+\mu \alpha}{1+\alpha}\right) \mathrm{W}(\mathrm{m})+\frac{\mu}{S(m)^{2} X(m)^{2}} S^{*}(m) X^{*}(m) E(m) \\
\mathrm{W}(\mathrm{m}+1)=\left(\frac{1}{1+\alpha}\right) \mathrm{W}(\mathrm{m})+\frac{\mu}{S(m)^{2} X(m)^{2}} S^{*}(m) X^{*}(m) E(m)
\end{gathered}
$$

Leakage coefficient $\gamma$ is,

$$
\begin{gathered}
\gamma=\frac{\alpha}{\mu(1+\alpha)} \\
\mu=\frac{\mu}{S(m)^{2} X(m)^{2}} \\
\mathrm{~W}(\mathrm{~m}+1)=(1-\mu \gamma) \mathrm{W}(\mathrm{m})+\mu S^{*}(m) X^{*}(m) E(m)
\end{gathered}
$$

Taking IFFT,

$$
\mathrm{W}(\mathrm{m}+1)=W(m)+\mu I F F T\left\{\frac{S(m) X^{*}(m) E(m)}{S(m)^{2} X(m)^{2}}-\Gamma(\mathrm{m}) \mathrm{W}(\mathrm{m})\right\}
$$

Where,

Input power,

$$
\Gamma(\mathrm{m})=\operatorname{diag}\left[\gamma_{0}(\mathrm{~m}), \gamma_{1}(\mathrm{~m}), \ldots \ldots \ldots \cdot \gamma_{2 N-1}(\mathrm{~m})\right]
$$

$$
\begin{gathered}
\mathrm{P}(\mathrm{m})=\mathrm{E}\left\{X(m)^{2}\right\} \\
\mathrm{P}(\mathrm{m})=\mathrm{P}(\mathrm{m}-1)(-\beta)\left[X(m) X^{*}(m)\right]
\end{gathered}
$$

\section{Power constrained algorithm}

In some applications, the filter output power is to be limited, for that the gain coefficient constraint is replaced by an output power constraint. Better frequency performance can be achieved by estimating the power in each frequency level, making the algorithm more selective in attenuating those frequencies in violation of the constraint.

Cost function is given by,

$$
\mathrm{P}(\mathrm{m}+1)=\frac{1}{N} \sum_{k=0}^{N-1} W(m+1)^{2} X(m)^{2}
$$

$$
\begin{aligned}
& J(m+1)=\delta W(m+1)^{2}+ \operatorname{Re}\left\{\lambda^{*}[D(m)-S(m) W(m+1) X(m)]\right\}+\alpha\left[[\mathrm{P}(\mathrm{m}+1)-\mathrm{C}]^{2}\right] \\
& \alpha=2 \alpha \mathrm{X}(m)^{2}[P(m)-C] \\
& P(m+1)=W(m+1)^{2} P(m+1) \\
& J(m+1)=\delta W(m+1)^{2}+ \operatorname{Re}\left\{\lambda^{*}[D(m)-S(m) W(m+1) X(m)]\right\}+\alpha\left[[\mathrm{P}(\mathrm{m}+1)-\mathrm{C}]^{2}\right]
\end{aligned}
$$

Repeating the earlier equations,

Where,

$$
\mathrm{W}(\mathrm{m}+1)=W(m)+\mu I F F T\left\{\frac{S^{*}(m) X^{*}(m) E(m)}{S(m)^{2} X(m)^{2}}-\Gamma(\mathrm{m}) \mathrm{W}(\mathrm{m})\right\}
$$

$$
\Gamma(\mathrm{m})=\operatorname{diag}\left[\gamma_{0}(\mathrm{~m}), \gamma_{1}(\mathrm{~m}), \ldots \ldots \ldots \gamma_{(2 N-1)}(\mathrm{m})\right]
$$

\section{Convergence analysis}

Here all the signals are assumed as white, zero-mean, Gaussian wide-sense stationary, and employ the independence assumption under a steady-state condition, where the constraint violation is constant and the transform-domain weights are mutually uncorrelated. A normalized input power of unity is also used, which then allows the analysis to apply to both gain-constrained and power constrained cases. 
Expectation result is given by,

$$
\begin{gathered}
\mathrm{E}[\mathrm{W}(\mathrm{m}+1)]=[1-\mu(\gamma+1)] \mathrm{E}[\mathrm{W}(\mathrm{m})]+\mu W \\
\lim _{m \rightarrow \infty} E[W(m)]=\mu W \sum_{i=0}^{m-1}[1-\mu(\gamma+1)]^{\mathrm{m}-1-\mathrm{i}} \\
W=\frac{W}{1+\gamma}
\end{gathered}
$$

Deviation from steady state,

$$
\begin{gathered}
V(m)=W(m)-W \\
V(m+1)=\left[1-\mu \gamma-\mu X^{*}(m) X(m)\right] V(m)+\mu \eta \mathrm{X}(\mathrm{m})-\mu \gamma W \\
E[\mathrm{~V}(\mathrm{~m}+1)]=[1-\mu(\gamma+1)] \mathrm{E}[\mathrm{V}(m)- \\
|1-\mu(\gamma+1)|<1 \\
\mu<\frac{2}{1+\gamma} \\
E\left[\mathrm{~V}(\mathrm{~m}+1) \mathrm{V}^{*}(\mathrm{~m}+1)\right]=\mathrm{E}\left[(1-\mu \gamma-\mu)^{2} V(m) V^{*}(m)\right]+\frac{\mu^{2} \sigma^{2}}{X(m)^{2}}+\mu^{2} \gamma^{2} \mathrm{~W} W^{*}-\mu \gamma E[(1-\mu \gamma- \\
\left.\mu) V(m) W^{*}\right]-\mu \gamma E\left[(1-\mu \gamma-\mu) V^{*}(m) W\right]
\end{gathered}
$$

State space model is given by,

Where,

$$
Z(m+1)=A Z(m)+B
$$

The maximum constraint violation is given by,

$$
\begin{gathered}
\mathrm{A} 11=1-2 \mu(\gamma+1)+\mu^{2}\left(\gamma^{2}+2 \gamma+1\right) \\
\quad\left|1-2 \mu(\gamma+1)+\mu^{2}\left(\gamma^{2}+2 \gamma+1\right)\right|<1
\end{gathered}
$$

\section{Comparison}

The improved convergence performance of the CLD algorithm as compared to the CSD algorithm and the leaky LMS in both gain-constrained and power-constrained applications is shown. The constraint terms $\mathrm{C}, \alpha$ can be held as constants in the simulations, but could be shaped over frequency for specific applications. External uncorrelated Gaussian white noise with a variance of 0.01 is added for the convergence comparisons, and an average of 100 runs is plotted.

\subsection{Gain constrained algorithm}

Using a unity gain secondary path, a 3-dB coefficient gain constraint is imposed. The CLD algorithm has the fastest convergence performance. The CSD algorithm began converging in a similar manner, but was not able to fully achieve the relatively high $20 \mathrm{~dB}$ gain required at the lowest frequencies. However, other simulations without deep secondary path nulls showed that the two algorithms converge to similar final weight values, with the CLD having a faster convergence rate.

\subsection{Power constrained algorithm}

The new CLD algorithm reduces the output effectively in the frequencies of power-constraint violation, while minimizing the effect at other frequencies when compared with the other algorithms. The new CLD algorithm reduces the output in the frequencies of power-constraint violation, while minimizing the effect at other frequencies.

\section{Conclusion}

The new CLD algorithm provides faster convergence and improved frequency response performance, especially in coloured noise environments. Additionally, the new CLD algorithm has the ability to handle multiple constraints in both gain-constrained and power- constrained applications. 


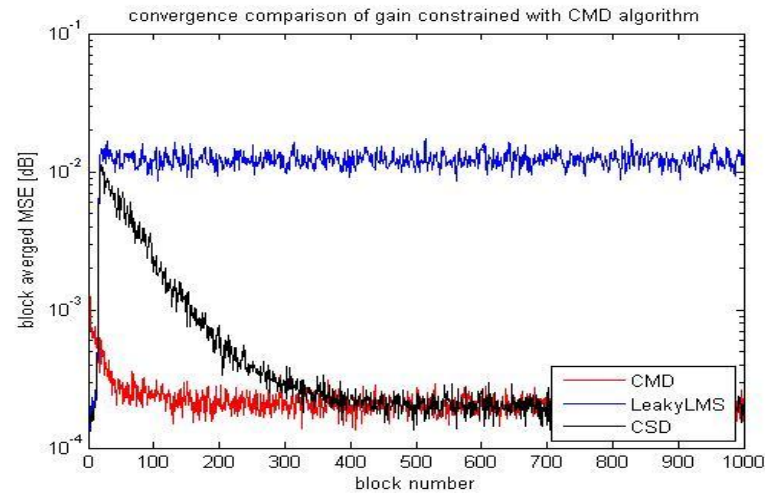

Figure 2 convergence comparison of gain constrained algorithms

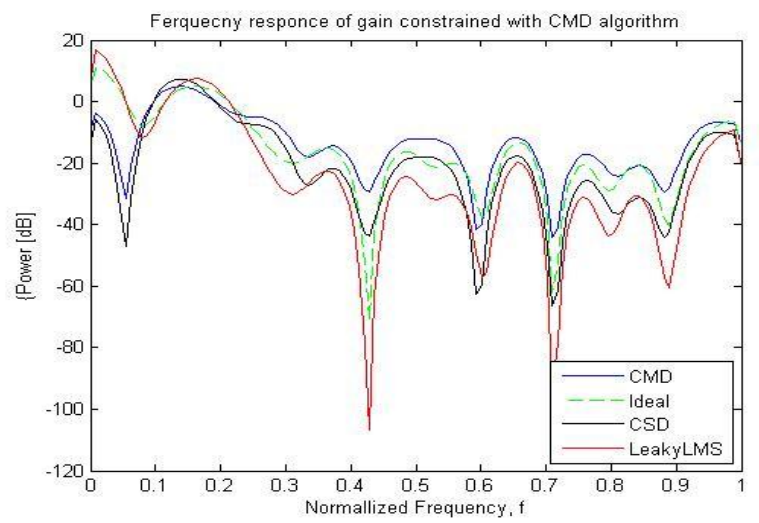

Figure 3 Comparison of Frequency response of gain constrained algorithms

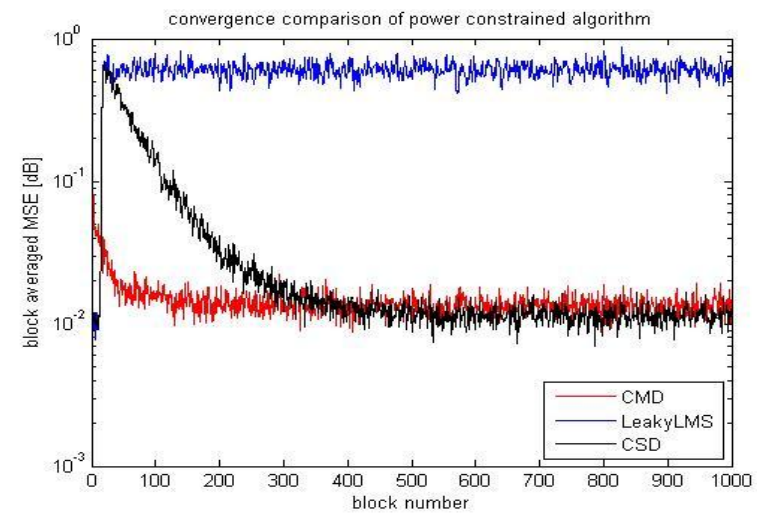

Figure 4 Convergence comparison of power constrained algorithms

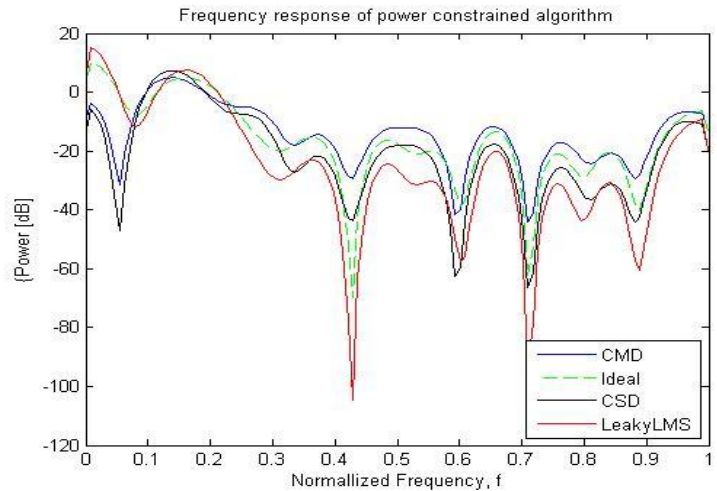

Figure 5 Comparison of Frequency response of power constrained algorithms 


\section{Future Scope}

The future scope of this idea can improve the convergence rate even more than existing rate. The determination of the exact convergence point can be improved to reduce the convergence time and thus the speed can be enhanced.

\section{References}

[1]. Romain Serizel, Marc Moonen,Jan Wouters, and Søren Holdt Jensen, “A Zone-of-Quiet Based Approach to Integrated Active Noise Control and Noise Reduction for Speech Enhancement in Hearing Aids”, in IEEE Trans. Signal Process. 20(6), 15587916(2012)

[2]. Muhammad Tahir Akhtar and Wataru Mitsuhashi, "Improving Performance of Hybrid Active Noise Control Systems for Uncorrelated Narrowband Disturbances", in IEEE Trans.Signal Process. 19(7), 1558-7916(2011)

[3]. Roberto Napoli and Luigi Piroddi, "Nonlinear Active Noise Control With NARX Models", in IEEE Trans. Signal Process.18 (2), 1558-7916(2010)

[4]. Liang Wang and Woon-Seng Gan, "Convergence Analysis of Narrowband Active Noise Equalizer System Under Imperfect Secondary Path Estimation”, in IEEE Trans. Signal Process. 17(4), 1558-7916(2009)

[5]. Yegui Xiao, Liying Ma and Koji Hasegawa, "Properties of FXLMS-Based Narrowband Active Noise Control With Online Secondary-Path Modeling”, in IEEE Trans. Signal Process. 57(8), 1053-587X (2009)

[6]. E. Prashanth Reddy, Debi Prasad Das, and K. M. M. Prabhu, "Fast Adaptive Algorithms for Active Control of Nonlinear Noise Processes", in IEEE Trans. Signal Process. 56(9), 1053-587X (2009)

[7]. Alberto Carini and Silvia Malatini, and Silvia Malatini, "Optimal Variable Step-Size NLMS Algorithms With Auxiliary Noise Power Scheduling for Feedforward Active Noise Control” in IEEE Trans. Signal Process. 16(8) 1558-7916 (2008)

[8]. Miguel Ferrer, Alberto Gonzalez, Maria de Diego and Gema Piñero, "Fast Affine Projection Algorithms for Filtered-x Multichannel Active Noise Control", in IEEE Trans. Signal Process. 16(8) 1558-7916 (2008)

[9]. D.P. Das, G. Panda and S.M. Kuo, "New block filtered-X LMS algorithms for active noise control systems", in IEEE Trans. Signal Process. 1(8) 1558-7916 (2007)

[10]. Jiashu Zhang' and Heng-Ming Tai2, "Adaptive Noise Cancellation Algorithm for Speech Processing”, in IEEE Trans. Signal Process. 5(18) 4244-0783 (2007) 